\title{
BMJ Open Comparison of outcomes of neurosurgical operations performed before and during the COVID-19 pandemic: a matched cohort study
}

\author{
Emma Toman (D) , ${ }^{1,2}$ Wai Cheong Soon, ${ }^{3}$ Gopiga Thanabalasundaram, ${ }^{2}$ \\ Daniel Burns, ${ }^{4,5}$ Vladimir Petrik, ${ }^{2}$ Colin Watts, ${ }^{2,6}$ Victoria Wykes, ${ }^{2,7}$ Anwen White ${ }^{2}$
}

To cite: Toman E, Soon WC, Thanabalasundaram G, et al. Comparison of outcomes of neurosurgical operations performed before and during the COVID-19 pandemic: a matched cohort study. BMJ Open 2021;11:e047063. doi:10.1136/ bmjopen-2020-047063

- Prepublication history for this paper is available online. To view these files, please visit the journal online (http://dx.doi. org/10.1136/bmjopen-2020047063).

Received 17 November 2020 Revised 04 February 2021 Accepted 08 February 2021
Check for updates

(c) Author(s) (or their employer(s)) 2021. Re-use permitted under CC BY-NC. No commercial re-use. See rights and permissions. Published by BMJ.

For numbered affiliations see end of article.

Correspondence to

Emma Toman;

ekt635@student.bham.ac.uk

\section{ABSTRACT}

Objective To determine how the first wave of the COVID-19 pandemic affected outcomes for all operatively managed neurosurgical patients, not only those positive for SARS-CoV-2.

Design Matched cohort (pairwise method).

Setting A single tertiary neurosurgical referral centre at a large UK Major Trauma Centre.

Participants During the first COVID-19 wave, 231 neurosurgical cases were performed. These cases were matched to cases from 2019. Cases were matched for age $( \pm 10$ years), primary pathology and surgical procedure. Cases were excluded from analysis if either the age could not be matched to within 10 years, or the primary pathology or procedure was too unique. After exclusions, 191 cases were included in final analysis.

Outcome measures Primary outcomes were 30-day mortality and postoperative pulmonary complications. Secondary outcomes included Glasgow Outcome Score (GOS) on discharge, length of stay (LoS), operative and anaesthetic times and grade of primary surgeon. An exploratory outcome was the SARS-CoV-2 status of patients.

Results There was no significant difference between the pandemic and matched cohorts in 30-day mortality, pulmonary complications, discharge GOS, LoS, operative or anaesthetic times. There was a significant difference in the variation of grade of primary surgeon. Only $2.2 \%(n=5)$ of patients had a SARS-CoV-2 positive swab.

Conclusion During the first UK wave of the COVID-19 pandemic, the mortality, morbidity and functional outcomes of operatively managed neurosurgical patients at University Hospitals Birmingham were not significantly affected compared with normal practice. The grade of primary surgeon was significantly more senior and adds to the growing body of evidence that demonstrates how the pandemic has negatively impacted UK surgical training. Mixing COVID-19 positive, unknown and negative cases did not significantly impact on outcomes and indicates that further research is required to support the implementation of evidence-based surgical pathways, such as COVID-light sites, throughout the next stage of the pandemic.

\section{Strengths and limitations of this study}

- This is the first study to individually match neurosurgical cases performed during the first pandemic wave to cases performed under 'pre-pandemic' conditions.

- Primary outcomes have been designed to be comparable with prior international surgical cohort studies.

- The numbers of patients analysed are small and from a single unit only.

- Not all neurosurgical cases performed during the first pandemic phase could be matched to prepandemic cases.

- Given the evolving understanding of the virus during the first wave, there was no universal swabbing protocol that could be applied to all cases in the pandemic cohort.

\section{INTRODUCTION}

\section{Background and rationale}

The first cases of COVID-19 were diagnosed in the UK at the end of January $2020 .{ }^{1}$ Hospitals began to prepare for the anticipated influx of severely unwell patients and surgical departments were advised to adapt their protocols, create additional capacity and protect their vulnerable patient groups. By mid-March, neurosurgical elective procedures were cancelled at the University Hospitals Birmingham (UHB) Foundation Trust. Exceptions were cancer, life-limiting cases or cases that ran a high risk of significant deterioration within 2 weeks if left untreated. ${ }^{2}$ Neuro-theatre capacity was reduced to one theatre running 24 hours a day and an additional ad hoc emergency daytime list where neuro-anaesthetic support allowed. These changes were necessary as UHB became the Trust 'worst hit' by COVID- $19^{3}$ with the Office for National Statistics confirming that Birmingham had the highest peak excess mortality of any major British city at $249.7 \%{ }^{4}$ 
While there have been several studies published on the restructuring of neurosurgery services and impact of COVID-19 on workload, ${ }^{5-7}$ there is currently limited evidence as to the impact of COVID-19 on the outcomes that such changes have had for neurosurgical patients. In May 2020, the COVIDSurg collaborative determined that postoperative pulmonary complications occur in 50.0\% of neurosurgical patients with perioperative SARS-CoV-2 infection and 30-day mortality was $18.4 \% .^{8}$ A US group reported the incidence of COVID-19 in neurosurgical patients to be $5.4 \%$ with a higher rate of complications in positive patients compared with negative patients. ${ }^{9}$ While it is vital to gather evidence on how SARS-CoV-2 affects the outcome of infected neurosurgical patients, the majority of patients (almost 95\%) remain uninfected. ${ }^{9}$ There is currently no evidence to describe how the pandemic has affected the outcome of the entire neurosurgical cohort when compared with 'normal' neurosurgical practice.

This study compares the outcomes of patients undergoing neurosurgical procedures during the height of the pandemic against a matched cohort from prior to the COVID-19 outbreak.

\section{Objectives}

1. To compare the rates of 30-day mortality and pulmonary complications.

2. To compare functional outcome and length of hospital stay (LoS).

3. To compare anaesthetic and operative factors.

4. To determine rates of patients screened for COVID-19, incidence of confirmed cases and their clinical course.

\section{MATERIALS AND METHODS \\ Study design}

The study was undertaken at the Queen Elizabeth Hospital Birmingham (QEHB) as a single-centre, matched cohort study. The QEHB is the largest hospital within the UHB Foundation Trust, is one of three regional adult neurosurgical units in the West Midlands, and is responsible for a mixed urban and rural population.

\section{Participants}

Operations performed during the initial stage of the pandemic (19 March 2020-1 June 2020) were matched to pre-pandemic cases (11 March 2019-11 September 2019). The pandemic time period was chosen as this was the interval in which elective neurosurgery cases at UHB were cancelled and will be referred to as the 'initial pandemic phase' from here onwards. Cases were matched in a pairwise fashion for age at time of procedure $( \pm 10$ years $)$, primary pathology and procedure. Primary pathology included severity where appropriate; for example, poor grade subarachnoid haemorrhage (SAH) was matched with another SAH of equivalent poor grade. Procedure was also matched to site where significant; for example a left-sided frontal craniotomy was matched with another left-sided frontal craniotomy rather than a right-sided. Ward-based procedures such as lumbar drain and intracranial pressure bolt insertion were not included. Cases were deemed unmatchable and were excluded from analysis if either the age could not be matched to within 10 years, or the primary pathology or procedure was too unique.

\section{Procedures}

Cases were identified by performing a search of the Galaxy electronic operating theatre system for all operations carried out under a neurosurgical consultant within the chosen time periods. Galaxy and the Prescribing Information and Communications System were then used to collate relevant variables.

Demographic data included age and gender. Operative data included name of procedure, grade of primary surgeon and operative time points. Grade of surgeon was defined as junior Senior Registrar (SpR) (ST3-5 or equivalent), senior SpR (ST5-8 or equivalent), fellow or consultant. Preoperative anaesthetic time was defined as time 'into anaesthetic room' to 'knife to skin', operative time was defined as 'knife to skin' to 'skin closure' and postoperative anaesthetic time was defined as 'skin closure' to time 'into theatre recovery'.

Clinical data included LoS in days, discharge Glasgow Outcome Score (GOS),${ }^{10}$ 30-day mortality, postoperative pulmonary complications and COVID-19 swab status. Swabs were deemed to be positive if SARS-CoV-2 RNA was detected by the standard RT-PCR laboratory test.

COVID-19 swabbing protocols were constantly evolving during this early phase of the pandemic as scientific knowledge and testing increased. For this reason, there is no one single consistent swabbing protocol for the time period interrogated in this study and as such reflects 'reallife' practice.

\section{Outcomes}

Primary outcomes

1. 30-day mortality.

2. Postoperative pulmonary complication.

Primary outcomes were designed to be comparable with the COVIDSurg study. ${ }^{8}$ For 30-day mortality, day of surgery was treated as day 0 . Postoperative pulmonary complication was defined as pneumonia, acute respiratory distress syndrome or unexpected postoperative ventilation (any episode of non-invasive ventilation, invasive ventilation or extracorporeal membrane oxygenation after initial extubation after surgery; or patient could not be extubated as planned after surgery).

\section{Secondary outcomes}

1. Discharge GOS. ${ }^{1-5}$

2. LoS in hospital (days).

3. Grade of primary surgeon.

4. Preoperative and postoperative anaesthetic time (minutes).

5. Operative time (minutes).

The GOS is an objective, functional outcome score from 1 to $5 .{ }^{11}$ Favourable GOS was defined as a score of 
4 (moderate disability) or 5 (good recovery). Unfavourable GOS was defined as a score of 1 (dead), 2 (vegetative state) or 3 (severe disability).

\section{Exploratory outcome}

COVID-19 status both preoperatively and postoperatively and the clinical course of positive patients.

\section{Statistical methods}

The study was conducted and reported in line with Strengthening the Reporting of Observational Studies in Epidemiology guidelines for cohort studies. ${ }^{12}$ Continuous data were tested for normal distribution prior to analysis. Parametric data are presented as mean with $95 \%$ CI, and the unpaired t-test used to detect differences between groups. Non-parametric data are presented as median with IQR, and Mann-Whitney used to detect differences between groups. The $\mathrm{X}^{2}$ test was used for categorical data. Missing data points were removed from final analyses. Clinical significance was defined as $\mathrm{p}<0.05$.

Analyses were performed using GraphPad Prism software V.8.4.3 for Windows.

\section{Patient and public involvement}

While there has been significant patient and public involvement (PPI) with regard to patient pathways during the pandemic, there was no specific PPI consultation for this study. This was due to the acutely evolving nature of the pandemic, time limits and manpower restraints.

\section{RESULTS}

Overall, 231 cases were performed during the initial pandemic phase. Of these $17.3 \%(n=40)$ were unmatchable and so were removed from the final analysis of primary and secondary outcomes. A final cohort of 191 cases from the initial pandemic phase were successfully matched to 191 cases from 2019.

The mean age for patients in the pandemic group was 55 years and the matched group, 54 years. There was no statistically significant difference between the age of the two cohorts $(\mathrm{p}=0.795$ (95\% CI -3.7 to 2.8$)$ ). With regard to gender, $42.4 \%(\mathrm{n}=81)$ and $47.6 \% \quad(\mathrm{n}=91)$ were women in the pandemic and matched cohorts, respectively. There was no statistically significant difference in gender between the two groups $(\mathrm{p}=0.304)$.

In terms of theatre location, during the pandemic $97.4 \% \quad(\mathrm{n}=186)$ of cases were performed in a dedicated emergency neurosurgical theatre and 2.6\% ( $n=5)$ performed in a shared-specialties emergency theatre. Cases from the matched cohort were performed across six separate neurosurgery theatres.

\section{Primary outcomes}

There was no statistically significant difference in either 30-day mortality or rates of postoperative pulmonary complication between the pandemic and matched cohorts (see table 1 ).
Table 1 Comparison of primary outcomes and discharge GOS

\begin{tabular}{lccl}
\hline & $\begin{array}{l}\text { Pandemic } \\
(\mathbf{n}=191)\end{array}$ & $\begin{array}{l}\text { Matched } \\
(\mathbf{n}=191)\end{array}$ & P value \\
\hline $\begin{array}{l}\text { 30-day mortality } \\
\text { Postop pulmonary } \\
\text { complications }\end{array}$ & $3.7(7)$ & $3.1(6)$ & 0.778 \\
$\begin{array}{l}\text { Favourable outcome } \\
\text { (GOS 4-5) }\end{array}$ & $89.9(170)^{*}$ & $92.1(176)$ & 0.366 \\
$\begin{array}{l}\text { Unfavourable outcome } \\
\text { (GOS 1-3) }\end{array}$ & $10.4(20)^{*}$ & $7.9(15)$ & \\
\hline
\end{tabular}

Data are presented as \% (n).

*One patient still an inpatient at time of analysis and was not included in analysis.

GOS, Glasgow Outcome Score.

\section{Secondary outcomes}

The majority of patients in both groups were discharged with a favourable GOS: $89.9 \%(n=170)$ and $92.1 \%(n=176)$ for pandemic and matched cohorts, respectively. There was no statistically significant difference in discharge GOS between the pandemic and matched cohorts (see table 1 and figure 1).

Median LoS was 4.5 days (IQR 2.0-10.3) for the pandemic cohort and 6.0 days (IQR 2.0-18.0) for the matched cohort. There was no statistically significant difference in LoS between pandemic and matched cohorts $(\mathrm{p}=0.25)$.

There was no statistically significant difference in preoperative anaesthetic time, operative time or postoperative anaesthetic time between pandemic and matched cohorts (see table 2). However, there was a significant difference in the variation of grade of primary surgeon between the two cohorts. Although the number of cases that consultants performed remained the same, there was a trend towards more cases being performed by senior SpRs and fellows rather than junior SpRs during the pandemic (see table 2).

\section{COVID-19}

For this section, all 231 participants for the pandemic period were analysed, including the unmatched cases. With regard to COVID-19 testing, 60.1\% ( $\mathrm{n}=139)$ were swabbed preoperatively and $37.2 \% \quad(n=86)$ were swabbed postoperatively; some were swabbed both preoperatively and postoperatively. Twenty-seven per cent $(n=63)$ of cases had neither preoperative nor postoperative swabs. Five patients in total tested positive for COVID-19 (see table 3 for case details). Four patients with a positive COVID-19 swab were operated on in the same theatre.

\section{DISCUSSION}

This study has demonstrated that the mortality and morbidity of neurosurgical patients undergoing operative management at UHB was not affected by the initial 


\section{Discharge GOS}

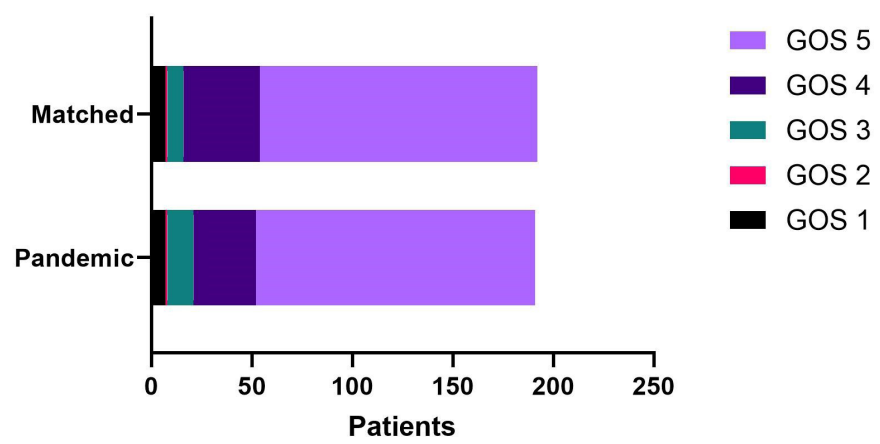

Figure 1 Comparison of discharge GOS. GOS, Glasgow Outcome Score.

COVID-19 outbreak. The patient selection, preoperative, perioperative and postoperative protocols at UHB for neurosurgery during the pandemic have ensured the short-term outcome for patients has not significantly changed compared with normal practice. During the pandemic, all neurosurgical consultants and registrars who were part of the on-call rota underwent fit testing for FFP3 masks. When operating on patients with unknown or pending COVID-19 status, all theatre staff wore FFP3 mask, protective face-shield/visors or eye-goggles perioperatively. When operating on patients with negative COVID-19 result, there was no requirement to wear an FFP3 mask. However, during intubation and extubation in all cases, the anaesthetic team had full personal protective equipment (PPE) due to high risk of exposure to aerosol. In all transsphenoidal surgery cases, all theatre staff wore PPE perioperatively. Such protocols and practices should therefore be reinstated in future pandemic waves.

Discussion surrounding how these results may impact on practice during this recovery phase of the pandemic is more complex. Current guidelines from both the National Institute of Health and Care Excellence (NICE) and the Royal College of Surgeons England (RCS) recommend strict social distancing, swabbing regimes and the separation of COVID-19 positive and negative patients. ${ }^{13}{ }^{14}$ The RCS goes further by stating that every hospital should have access to 'COVID-light sites' that 'might be created at independent hospitals, within designated areas in National Health Service (NHS) hospitals or for an entire hospital functioning as an NHS network hub'. ${ }^{15}$ A recent survey however demonstrated that nationally $26 \%$ of hospitals have not been able to access COVID-light facilities. ${ }^{16}$

To comply with NICE and RCS guidance, UHB designed 'hot' and 'cold' surgical pathways dependant on preoperative COVID-19 status. This new strategy was implemented on 1 June 2020 when elective operating restarted. Designated hot and cold pathways will increase public confidence that the risk of transmission is being reduced as much as is physically possible. The downside to having separate pathways is the huge logistical organisation they require, the associated financial impact of running additional swabbing, admission and surgical pathways, and a greatly reduced theatre capacity at a time where waiting lists are the highest they have been for over a decade. ${ }^{17}$

This study is the first to demonstrate that mixing COVID-19 positive, unknown and negative cases in a single large centre has not impacted patient outcomes during the initial phase of the pandemic. Our data suggest that it is not necessary to provide 'COVID-light centres', and as long as rigorous protocols are adhered to, neurosurgical care can proceed with acceptable healthcare outcomes comparable with pre-pandemic practice. While this small study does not definitively prove that mixing of patients is safe on a national scale, it certainly highlights that more investigation is required to support or refute these findings. The authors therefore strongly recommend that a larger, multicentre study should be undertaken during the next stage of the pandemic to assess whether outcome and infection rate is truly improved by such a costly and time-intensive approach to elective surgery.

\begin{tabular}{|c|c|c|c|}
\hline & Pandemic & Matched & \\
\hline & $n=191$ & $n=191$ & $P$ value \\
\hline Grade of primary surgeon & & & 0.0014 \\
\hline Junior SpR & $5.2(10)$ & $6.3(12)$ & \\
\hline Senior SpR & $7.3(14)$ & $14.7(28)$ & \\
\hline Fellow & $9.4(18)$ & $1.6(3)$ & \\
\hline Consultant & 78.0 (149) & $77.5(148)$ & \\
\hline Preop anaesthetic time & $58.5(44.0-76.0)$ & $57(46.0-72.0)$ & 0.33 \\
\hline Operative time & $101.5(57.0-150.5)$ & $102(54.0-148.0)$ & 0.792 \\
\hline Postop anaesthetic time & $19(11.75-28.0)$ & $17(11.0-24.0)$ & 0.113 \\
\hline
\end{tabular}

Data are presented as \% (n) for grade of primary surgeon and median (IQR) for anaesthetic and operative times. Units of time presented are minutes.

$\mathrm{SpR}$, Senior Registrar. 
Table 3 Details of COVID-19 positive patients

\begin{tabular}{lllllll}
\hline Patient & $\begin{array}{l}\text { Preop CoVID-19 } \\
\text { swab }\end{array}$ & $\begin{array}{l}\text { Postop } \\
\text { CoVID-19 } \\
\text { swab }\end{array}$ & $\begin{array}{l}\text { Postop pulmonary } \\
\text { complication }\end{array}$ & $\begin{array}{l}\text { 30-day } \\
\text { mortality }\end{array}$ & $\begin{array}{l}\text { Length } \\
\text { of stay }\end{array}$ \\
\hline A & Positive & Positive & Pneumonia & Survived & 4 & 23 \\
B & Positive & Positive & Pneumonia & Survived & 4 & 23 \\
C & Not swabbed & Positive & Nil & Survived & 5 & 22 \\
D & Negative & Positive & Nil & Survived & 5 & 6 \\
E & Negative & Positive & Unexpected postop & Survived* & 1 & RIP day \\
& & & ventilation & & & 42 \\
\hline
\end{tabular}

${ }^{*}$ Patient died at day 42 following admission (day 38 postop); immunocompromised individual with COVID-19 pneumonia listed as contributory factor as cause of death.

GOS, Glasgow Outcome Score.

It may seem intuitive that by increasing the amount of PPE anaesthetic and surgical teams must wear, the anaesthetic and operative time should also increase. Unpublished data from UHB analysing neuro-oncology operations during the first month of initial phase of the pandemic suggested that the average length of operation was significantly increased. However, our study has demonstrated that there was no significant difference in the length of operation or anaesthetic when compared with normal practice. It must be noted however that although actual times for anaesthetic and procedure have not been impacted, turnaround time between cases has anecdotally increased at UHB. This is due to an additional 15-minute theatre ventilation air change prior to each new case, supplementary cleaning protocols and the removal of the communal 'holding bay' prior to surgery. These data were not available retrospectively and so should be included in any future prospective study when analysing length of theatre time and planning realistic theatre lists.

The only statistically significant finding in this study was a difference in the grade of primary surgeon. Although there was no difference in the proportion of patients operated on by a consultant, there was a shift towards seniority among operations performed by nonconsultants. During the pandemic, more operations were primarily performed by senior SpRs and fellows than junior SpRs compared with normal practice. One reason for this may be that initially, more junior SpRs and senior house officers were redeployed to Intensive Care Unit and proning teams compared with senior SpRs. These data add to the growing body of evidence that COVID-19 has negatively impacted on surgical training in the UK. ${ }^{18} 19$ A UK-wide survey of neurosurgery trainees revealed that fewer training cases were available due to cancellation of elective lists, surgical rotations were halted, examinations cancelled and nationally there was an increase in out-of-hours work during the height of the pandemic. ${ }^{20}$ Training is also a factor that must be considered in the recommencement of elective surgery ${ }^{15}$ and should be included in any future study addressing the recovery of surgical services.

In summary, the mortality, morbidity and functional outcome of patients undergoing neurosurgical operations at UHB have not been affected by the COVID-19 pandemic. To the best of the authors' knowledge, this is the first study to match neurosurgical cases from the initial pandemic phase to pre-pandemic cases. Most of the surgical-related evidence up until now has focused on outcomes in patients with confirmed COVID-19. We must not forget however that the majority of surgical patients do not have COVID-19 and it is imperative that outcomes are studied in this group to help design evidence-based surgical pathways for the remainder of this national and global health disaster. This study is based on data from a single centre and focuses on neurosurgery patients only. Given the variation in national practices, we would encourage individual hospitals and Trusts to analyse outcomes in the same way. This would provide larger datasets and could identify which protocols have been most effective. In particular, it is important to ascertain whether mixing positive, unknown and negative cases in a shared surgical pathway is detrimental. If not, the national implementation of COVID-light sites should be called into question as these currently limit patient flow in a time of waiting list crisis.

\section{Limitations}

All matched cohort studies recognise the limitations of matching cases as not all variables can be taken into account. For a COVID-19 study in particular, we recognise that ethnicity ${ }^{21}$ and body mass index ${ }^{22}$ are contributing factors to morbidity and mortality and these variables were not matched.

The sample size included in this analysis is relatively small and so may be underpowered. Larger studies and meta-analysis would provide further data to support or refute these findings.

During the pandemic period, the UHB swabbing protocol evolved and as a result was not consistent across the whole study period. While a true reflection of our 
clinical practice this, along with the small number of COVID-19 positive patients, means that it is unsuitable to perform inferential statistics on these data. However, the authors felt it important to include descriptive data for the COVID-19 positive patients to enable inclusion in any future meta-analysis or power calculations.

\section{CONCLUSION}

During the initial phase of the COVID-19 pandemic, the mortality, morbidity and functional outcomes of operatively managed neurosurgery patients were not significantly affected compared with normal practice. Although anaesthetic and operative time was not altered, the grade of primary surgeon was significantly more senior which is likely to have negatively impacted on training opportunities for junior surgeons. This adds to the growing body of evidence that surgical training in the UK has been significantly affected by the pandemic. Mixing COVID-19 positive, unknown and negative cases did not significantly impact the outcomes of neurosurgical patients and calls into question the need for COVID-light sites. Larger multicentre studies are needed to confirm or refute these findings and collect prospective data to support the implementation of evidence-based surgical pathways now that we are entering the next wave of the pandemic.

\section{Author affiliations}

${ }^{1}$ Institute of Inflammation and Ageing, University of Birmingham College of Medical and Dental Sciences, Birmingham, UK

${ }^{2}$ Department of Neurosurgery, University Hospitals Birmingham NHS Foundation Trust, Birmingham, UK

${ }^{3}$ Department of Neurosurgery, Queen Elizabeth Hospital, Birmingham, UK

${ }^{4}$ Department of Infectious Diseases and Tropical Medicine, University Hospitals Birmingham NHS Foundation Trust, Birmingham, UK

${ }^{5}$ Department of Medicine, Royal Centre for Defence Medicine, Birmingham, UK ${ }^{6}$ Institute of Cancer and Genomic Studies, University of Birmingham, Birmingham, UK

${ }^{7}$ Institute of Cancer and Genomics, University of Birmingham College of Medical and Dental Sciences, Birmingham, UK

Contributors Conception and design-ET, AW and VP. Acquisition, analysis and interpretation of data-ET, GT, WCS, DB, VW, CW, VP and AW. Drafting and revising the manuscript-ET, GT, WCS, DB, VW, CW, VP and AW. All authors approved the final draft of the manuscript prior to submission.

Funding The authors have not declared a specific grant for this research from any funding agency in the public, commercial or not-for-profit sectors.

\section{Competing interests None declared.}

Patient and public involvement Patients and/or the public were not involved in the design, or conduct, or reporting, or dissemination plans of this research.

Patient consent for publication Not required.

Provenance and peer review Not commissioned; externally peer reviewed.

Data availability statement Data are available in a public, open access repository. Extra data can be accessed via the Dryad data repository at http://datadryad.org/ with the doi:10.5061/dryad.q83bk3jgr.

Open access This is an open access article distributed in accordance with the Creative Commons Attribution Non Commercial (CC BY-NC 4.0) license, which permits others to distribute, remix, adapt, build upon this work non-commercially, and license their derivative works on different terms, provided the original work is properly cited, appropriate credit is given, any changes made indicated, and the use is non-commercial. See: http://creativecommons.org/licenses/by-nc/4.0/.

\section{ORCID iD}

Emma Toman http://orcid.org/0000-0003-2142-1923

\section{REFERENCES}

1 Moss P, Barlow G, Easom N, et al. Lessons for managing highconsequence infections from first COVID-19 cases in the UK. Lancet 2020;395:e46.

2 Neurosurgery CSL. Email correspondence- cancellation of elective procedures. In: list Nd, editor, 2020.

3 Blanchard S. England's worst-hit hospitals: Data shows NHS body in Birmingham has recorded 820 coronavirus deaths as EVERY major trust has now counted at least one COVID-19 victim. Daily Mail 2020

4 Office for National Statistics. Comparisons of all-cause mortality between European countries and regions: January to June 2020 ONS, 2020. Available: https://www.ons.gov.uk/peoplepopulation andcommunity/birthsdeathsandmarriages/deaths/articles/comp arisonsofallcausemortalitybetweeneuropeancountriesandregions/ januarytojune2020

5 Fontanella MM, De Maria L, Zanin L, et al. Neurosurgical practice during the severe acute respiratory syndrome coronavirus 2 (SARS-CoV-2) pandemic: a worldwide survey. World Neurosurg 2020;139:e818-26.

6 Jean WC, Ironside NT, Sack KD, et al. The impact of COVID-19 on neurosurgeons and the strategy for triaging non-emergent operations: a global neurosurgery study. Acta Neurochir 2020;162:1229-40.

7 Hanrahan JG, Burford C, Adegboyega G, et al. Early responses of neurosurgical practice to the coronavirus disease 2019 (COVID-19) pandemic: a rapid review. World Neurosurg 2020;141:e1017-26.

8 COVIDSurg Collaborative. Mortality and pulmonary complications in patients undergoing surgery with perioperative SARS-CoV-2 infection: an international cohort study. Lancet 2020;396:27-38.

9 Dowlati E, Zhou T, Sarpong K, et al. In Reply to the Letter to the Editor Regarding "Case Volumes and Perioperative COVID-19 Incidence in Neurosurgical Patients During a Pandemic: Experiences at Two Tertiary Care Centers in Washington, DC". World Neurosurg 2020;143:633.

10 Jennett B, Bond M. Assessment of outcome after severe brain damage. Lancet 1975;1:480-4.

11 Jennett B, Bond M. Assessment of outcome after severe brain damage a practical scale. The Lancet 1975;305:480-4.

12 von Elm E, Altman DG, Egger M, et al. Strengthening the reporting of observational studies in epidemiology (STROBE) statement: guidelines for reporting observational studies. BMJ 2007;335:806-8.

13 NICE. Nice guideline NG-179. COVID-19 rapid guideline: arranging planned care in hospitals and diagnostic services: NICE, 2020. Available: https://www.nice.org.uk/guidance/ng179

14 RCS. Guidance for surgeons working during the COVID-19 pandemic Royal College of surgeons, 2020. Available: https://www.rcseng.ac. uk/coronavirus/joint-guidance-for-surgeons-v1/

15 RCS. Recovery of surgical services during and after COVID-19 Royal College of surgeons, 2020. Available: https://www.rcseng.ac. uk/coronavirus/recovery-of-surgical-services/

16 RCS. Survey findings: elective surgery during COVID-19 - Royal College of surgeons, 2020. Available: https://www.rcseng.ac.uk/ news-and-events/news/archive/survey-results-elective-surgeryunder-covid/

17 RCS. Surgeons call for 'COVID-negative' operating theatres across the UK to tackle 'hidden waiting list' - Royal College of Surgeons, 2020. Available: https://www.rcseng.ac.uk/news-and-events/mediacentre/press-releases/covid-negative-operating-theatres/

18 Khan KS, Keay R, McLellan M, et al. Impact of the COVID-19 pandemic on core surgical training. Scott Med J 2020;65:133-7.

19 Caruana EJ, Patel A, Kendall S, et al. Impact of coronavirus 2019 (COVID-19) on training and well-being in subspecialty surgery: a national survey of cardiothoracic trainees in the United Kingdom. $J$ Thorac Cardiovasc Surg 2020;160:980-7.

20 RCS. Royal College of Surgeons webinar "The effects of COVID-19 on UK neurosurgery", 2020.

21 Pan D, Sze S, Minhas JS, et al. The impact of ethnicity on clinical outcomes in COVID-19: a systematic review. EClinicalMedicine 2020;23:100404.

22 Pranata R, Lim MA, Yonas E, et al. Body mass index and outcome in patients with COVID-19: a dose-response meta-analysis. Diabetes Metab 2020:101178. 